\title{
A microscopic theory of low energy fission: fragment properties
}

W. Younes, D. Gogny, N. Schunck

January 14,2013

Fifth International Conference on Fission and Properties of Neutron Rich Nuclei Sanibel Island, FL, United States November 4, 2012 through November 10, 2012 
This document was prepared as an account of work sponsored by an agency of the United States government. Neither the United States government nor Lawrence Livermore National Security, LLC, nor any of their employees makes any warranty, expressed or implied, or assumes any legal liability or responsibility for the accuracy, completeness, or usefulness of any information, apparatus, product, or process disclosed, or represents that its use would not infringe privately owned rights. Reference herein to any specific commercial product, process, or service by trade name, trademark, manufacturer, or otherwise does not necessarily constitute or imply its endorsement, recommendation, or favoring by the United States government or Lawrence Livermore National Security, LLC. The views and opinions of authors expressed herein do not necessarily state or reflect those of the United States government or Lawrence Livermore National Security, LLC, and shall not be used for advertising or product endorsement purposes. 


\title{
A microscopic theory of low energy fission: fragment properties
}

\author{
W. Younes, D. Gogny, and N. Schunck \\ Lawrence Livermore National Laboratory \\ Livermore, $C A 94551$
}

\begin{abstract}
We present fully microscopic time-dependent calculations of fissionfragment properties (mass distributions, pre-scission energies, total kinetic and excitation energies) for the ${ }^{235} \mathrm{U}(n, f)$ and ${ }^{239} \mathrm{Pu}(n, f)$ reactions. The mass distributions for both reactions have been obtained as a function of incident neutron energy from thermal to $5 \mathrm{MeV}$. The various energies have been calculated for the thermal ${ }^{239} \mathrm{Pu}(n, f)$ reaction. We our calculations to experimental results, wherever possible.
\end{abstract}

\section{Introduction}

A goal and sensitive test of our understanding of fission at the microscopic level of interacting protons and neutrons is the development of a comprehensive theory of fission-fragment properties. We present a microscopic approach to the calculation of several crucial fission fragment properties (their mass yields, as well as their kinetic and excitation energies) within a single theoretical framework.

Ideally, we would follow the evolution of the full many body wave function of the parent nucleus as a function of time, and analyze it in terms of excitations built on top of the ground states of all possible fragments. The complexity of the many-body wave function makes this extremely difficult. Instead, we begin with a constrained Hartree-Fock-Bogoliubov (HFB) description of the parent nucleus, construct a wave packet from all HFB solutions with respect to a few collective coordinates relevant to the fission process, and allow the system to evolve in time out to scission. Thus we must necessarily recognize two regions within the space spanned by the collective coordinates: an inner region where the system is described by the Hamiltonian of the parent nucleus, and an outer region where the system is described by the Hamiltonian of the individual fragments, interacting essentially through their mutual Coulomb repulsion. These two regions are 
separated by a boundary that we identify as the set of scission points. Thus, two central concepts lie at the heart of this microscopic approach to fission: our definition of scission, and the choice of appropriate collective coordinate. We will elucidate these two concepts in this paper and use them in microscopic calculations of the fission-fragment properties for the ${ }^{235} \mathrm{U}(n, f)$ and ${ }^{239} \mathrm{Pu}(n, f)$ reactions.

\section{Theory}

How can we recognize scission? In standard HFB calculations for an actinide nucleus, with the quadrupole $\left(Q_{20}\right)$ and octupole $\left(Q_{30}\right)$ moments of nucleus under constraint, we can follow the evolution of the properties of the parent nucleus (e.g., its total energy, its hexadecapole moment Q40, its spatial density, etc) as a function of $Q_{20}$ and $Q_{30}$. In the $Q_{20}-Q_{30}$ space, we can usually identify a boundary line separating inner and outer regions where we observe a discontinuous change in various properties of the system, such as a marked drop in the total energy, and the transition of the spatial density from that of a single nucleus, to that of a pair of nuclei no longer connected by a neck. This abrupt transition, makes it impossible to recognize the fragment properties at scission. In order to identify the scission boundary more precisely, we have imposed the size of the neck ${ }^{1}$ separating the prefragments as an additional constraint. With this constraint, we can now look in detail at the individual quasiparticle states of the nucleus and assign them to either one of the two pre-fragments. Individual quasiparticles can be assigned to a particular pre-fragment based on whether most of its spatial density lies more on the side of that fragment, with respect to the position of the neck. Once this classification of quasiparticle states has been completed (with some allowance for weakly populated states that cannot easily be attributed to either pre-fragment), the interaction energy of the parent nucleus can be partitioned into separate contributions from each fragment, and a mutual (nuclear + Coulomb) interaction between them. We observe that the pre-fragments constructed by assigning individual quasiparticle states one at a time exhibit tails in their spatial distributions that stretch deeply into the complementary fragment. ${ }^{2,3}$ These tails can persist, even as the neck between the pre-fragments vanishes. Furthermore, our calculations for ${ }^{240} \mathrm{Pu}$ fission show that the mutual interaction energy between the prefragments varies linearly with the number of particles in those tails, and that each particle in the tails contributes $\sim 50 \mathrm{MeV}$ of attractive nuclear interaction energy between the pre-fragments. Under those conditions it becomes exceedingly difficult to define scission. 
This seemingly paradoxical state of affairs stems from the fact that all HFB calculations are defined only up to an arbitrary unitary transformation. ${ }^{4}$ We can choose this transformation to further localize the individual quasiparticle states on their respective pre-fragments and reduce the size of the tails, without changing any of the global properties of the HFB solution (total energy, moments, etc.) ${ }^{3}$ We can start to apply this transformation as soon as we recognize pre-fragments in the total density of the parent nucleus, and thereby follow the separation of the parent into its daughters progressively across the scission boundary. This localization procedure makes it possible to identify a physically meaningful scission point. Thus, in our recent letter ${ }^{3}$ we have adopted the following criteria to define scission, based on a microscopic analysis of the HFB solution after localization: 1) the Coulomb repulsion must greatly exceed the nuclear interaction between the pre-fragments (so that they will quickly fly apart), 2) the exchange interaction between the fragments must be small (so that the system behaves as two Bogoliubov vacuua as far as expectation values of interest are concerned), and 3) it is possible to excite two-quasiparticle states on each pre-fragment whose wave functions remain localized on that pre-fragment. With these criteria verified, the pre-fragments can be considered as separate entities (i.e. primary fragments) each with its own set of excitations, and interacting through a repulsive force acting only on their centers of mass. We emphasize that this description does not yet constitute a complete dynamical theory of scission, but it provides the crucial microscopic ingredients for such a theory.

Next, we discuss the choice of collective coordinates for the description of fission. We have found that $Q_{30}$ and the fragment mass are not in a oneto-one relationship. We have also previously alluded ${ }^{3}$ to the fact that, near scission, constraints on the properties of the individual pre-fragments are better suited to the description of the fission process. With that in mind, we introduce the mass asymmetry coordinate $\xi \equiv\left(A_{2}-A_{1}\right) / A$, where $A_{1}$ and $A_{2}$ are the numbers of particles (protons + neutrons) in each fragment, calculated as the integral of the total density of the parent nucleus to either side of the neck position, and $A=A_{1}+A_{2}$. We also define the separation distance $d$ between the centers of mass of the pre-fragments $d \equiv z_{2}-z_{1}$, where the $z_{i}$ are the centers of mass along the $z$ axis of symmetry of the parent nucleus. The positions $z_{i}$ are also obtained from integral moments of the total density to either side of the neck position. Although these new constraints rely on semi-classical definitions (i.e., sharp cuts in the total density to calculate integrals), the subsequent analysis of the scission 
points uses the quantum localization procedure discussed above and takes into account the effect of the pre-fragment tails.

With the microscopic definition of scission given above, and the choice of $\xi$ and $d$ as collective coordinates, we can calculate a large set of configurations that give a static picture of the fissioning system up to scission. We now complete this picture with a dynamic treatment of the fission process. This time-dependent description is needed to calculate the mass distribution of the fragments, as well as the partition of the energy available in fission between kinetic and excitation energy of the fragments. Therefore we need to derive a collective Hamiltonian that governs the evolution of the system. For this, we use a semiclassical approach, quantized a posteriori..$^{5,6}$ The time-dependent solution in the internal region in $(d, \xi)$ gives a flux across the scission boundary, which we interpret as the mass distribution of the fragments. The initial state for the time evolution depends on the initial excitation energy, and is constructed as in ref. ${ }^{6}$

At this point, we have given a fully microscopic description of the fission process in the interior region, up to scission. How do we connect the interior and exterior regions in the calculations? The interior region is described by adiabatic HFB calculations. If we continue to apply the same variational principle beyond scission, the ultimate result will invariably be two fragments infinitely far apart and in their ground states. However, our analysis of the interaction energy between pre-fragments suggests that the system breaks apart suddenly and that the fragments remain "frozen" in their configurations at that scission point. Beyond scission, we assume that the fragments propagate according to a Hamiltonian that depends only on their separation distance. We will need to estimate the kinetic energy of these fragments at scission, or pre-scission kinetic energy (PKE), within the context of our microscopic approach. The basic problem in the calculation of PKE is to estimate the "dissipation" of energy due to the coupling of the fission mode to other (transverse) degrees of freedom, such as intrinsic and collective excitations. Then, only motion in the fission direction will contribute to the PKE, which we estimate from the coupling between collective coordinates. We identify the fission direction at a scission point with the direction of maximum flux (also given by the direction of the probability current). We then calculate the flux $\phi$ in that direction, normalized by the squared amplitude $|g|^{2}$ of the wave function at this point in $(d, \xi)$ and observe that, for fission from many states at low excitation energies, this normalized flux is constant in time. This observation suggests a solution at scission that is a product of a local plane wave in the fission direction, 
and another function in the transverse direction which is cancelled out by the normalization of the flux. We now use the WKB approximation in the fission direction, in order to relate the normalized flux of the wave to its energy $E_{F}$ in that direction, $\phi /|g|^{2}=\sqrt{2 B_{F} E_{F}} / \hbar$, where $B_{F}$ is the inertia in the fission direction. In a one-dimensional model of fission which does not allow for transverse motion, the difference $\Delta E=E_{\mathrm{tot}}-V\left(d_{\mathrm{sc}}\right)$ between total energy of the parent nucleus in its initial state and at scission would appear entirely as PKE of the fragments. In general, the energy $E_{F}$ we calculated above will be smaller: $E_{F}<\Delta E$, with the difference being lost to transverse motion. We interpret $E_{F}$ as the PKE. With this last result, we can now connect the interior and exterior regions in the calculation. To establish this connection we invoke the conservation of total energy of the system: since the separation distance $d$ is the only coordinate in the exterior, and since $\Delta E-E_{F}$ is the energy in the direction transverse to $d$, we cannot associate it with the kinetic energy of the fragments, and therefore we assign it to their excitation energy. Note that we do not yet have a mechanism to describe this transfer in energy (although strides are being made in this direction ${ }^{7}$ ); this is only a model to estimate the energy "dissipated" due to the coupling between collective degrees of freedom.

\section{Discussion}

We have used the microscopic approach to calculate the mass distribution of fission fragments for the ${ }^{235} \mathrm{U}(n, f)$ and ${ }^{239} \mathrm{Pu}(n, f)$ reactions, as a function of incident neutron energy with $E_{n}=$ thermal to $5 \mathrm{MeV}$. For a given excitation energy of the compound fissioning nucleus, calculated fluxes from initial states within $+-500 \mathrm{keV}$ of that excitation energy were averaged together. We also account for fluctuations in particle number of the fragments due to pairing by applying a Gaussian smoothing to the calculated yields with a width suggested by the fluctuation in particle number.

We compare in Fig. 1 the calculated mass distributions for the ${ }^{235} \mathrm{U}(n, f)$ reaction before neutron emission, to experimental results from Straede et al. ${ }^{8}$ About $2 / 3$ of the calculated fragments yields lie within $30 \%$ of the experimental values, with the most significant discrepancies occurring near peak for thermal fission $\left(E_{n}=0.0 \mathrm{MeV}\right)$, and near symmetric fission $(A$ $=118$ ) for $E_{n} \geq 2 \mathrm{MeV}$. Similarly, we show in Fig. 1 the calculated mass distributions for the ${ }^{239} \mathrm{Pu}(n, f)$ reaction. In this case, experimental values for the pre-neutron yields are only available for $E_{n}=$ thermal. $^{9}$ At higher energies, we compare our microscopic calculations to the phenomenological general fission model (GEF) of Schmidt et al. ${ }^{10}$ At thermal energies, about 
$3 / 4$ of the calculated yields agree with experimental values to within $30 \%$. The agreement between our calculations and the data is comparable to that achieved by the phenomenological GEF model.

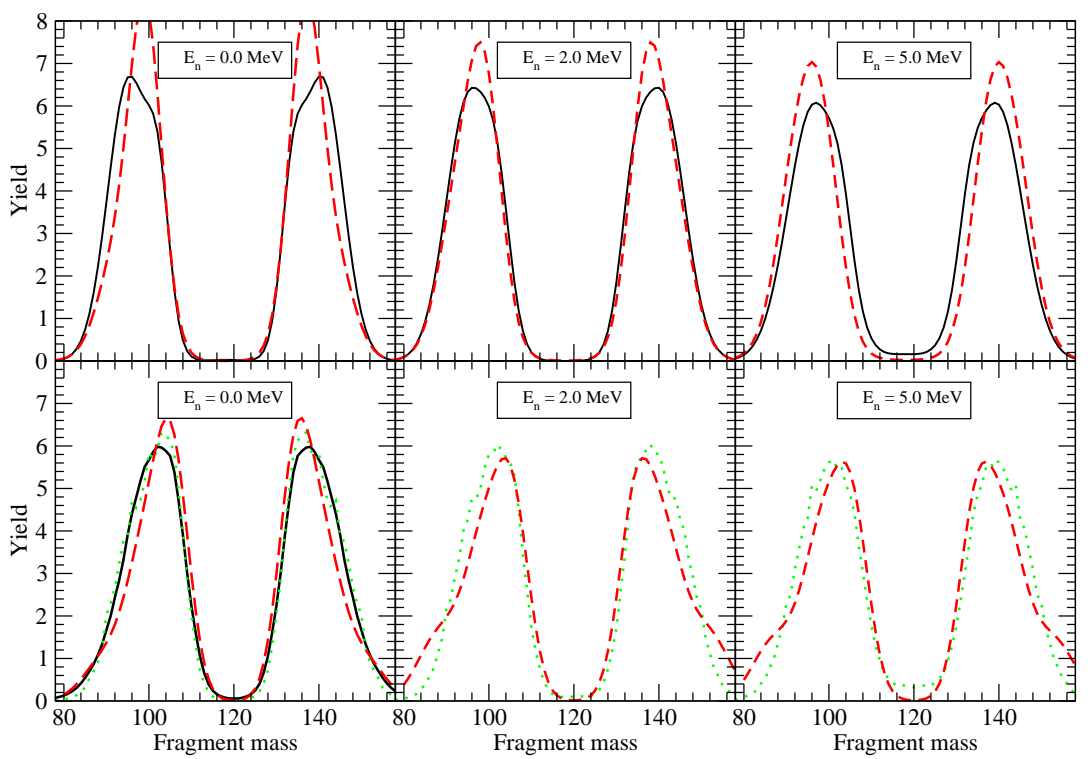

Fig. 1. Theoretical calculations of mass yields, before neutron emission, for ${ }^{235} \mathrm{U}(n, f)$ (top 3 panels) and ${ }^{239} \mathrm{Pu}(n, f)$ (bottom 3 panels), for different incident energies $E_{n}$. Microscopic-theory values (dashed lines) are compared to experimental data (solid curves) where available. the phenomenological model GEF of Schmidt et al. (dotted line) is also shown for ${ }^{239} \mathrm{Pu}(n, f)$.

Next, we apply the microscopic approach to the calculation of the fragment total kinetic (TKE) and total excitation (TXE) energies. Using the analysis of the normalized flux at scission described in the theory section, we have calculated $~ 50 \%$ of the saddle-to-scission energy going into PKE for thermal fission in the most probable fission mode. This result is in line with the assumption we made in our letter ${ }^{3}$ and is consistent with data-based estimates made by other authors. ${ }^{11}$ These PKE can be added to static calculations of the fragment kinetic and excitation energies at scission. With the quasiparticle states localized on the fragments, the kinetic energy at scission is calculated as the Coulomb interaction between the proton states 
in the complementary fragments. The fragment excitation energies at scission are obtained as the difference between their internal energies at scission and in their ground state. The ground state of the fragments was obtained by relaxing the constraint on the size of the neck joining them, and allowing the system to relax into its minimum-energy state. The TKE and TXE obtained for ${ }^{239} \mathrm{Pu}\left(n_{\mathrm{th}}, f\right)$ is compared in Fig. 2 to experimental values. The agreement between microscopic calculation and experiment is very good for the TKE, while the calculated TXE are low compared to experiment. We found the quality of this agreement was not significantly improved when we assumed a 70/30 split for the pre-scission excitation-to-kinetic energy ratio, compared to the 50/50 split assumed in Fig. 2. We also obtained similar agreement with data for the TKE and TXE when we used the D1M parametrization ${ }^{12}$ of the finite-range nucleon interaction, instead of the D1S values used in Fig. 2.

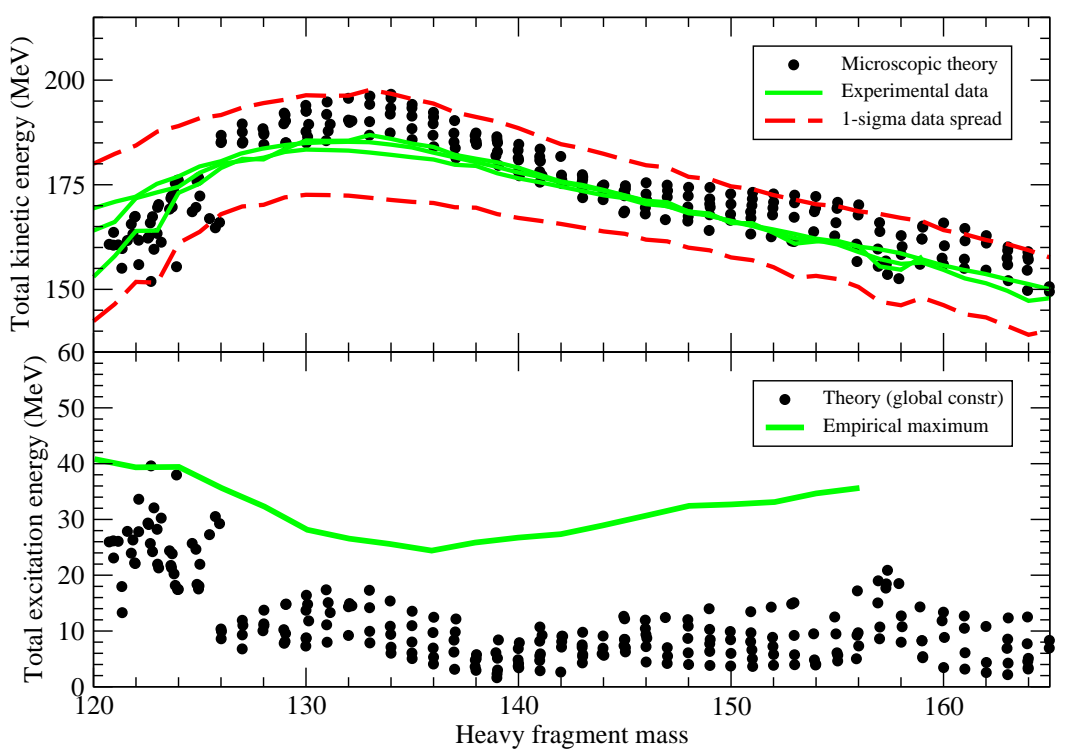

Fig. 2. Comparison of calculated and experimental TKE (top panel) and TXE (bottom panel) for the ${ }^{239} \mathrm{Pu}(n, f)$ reaction. 


\section{Conclusion}

We have described within a single microscopic framework several important aspects of fission: the mass distribution, pre-scission energy, and the total kinetic and excitation energies of the fragments. We obtain reasonable agreement with experiment, however discrepancies remain, especially in the calculation of the excitation energies of the fragments. These latter results can certainly be ameliorated, in particular with an improved calculation of the fragment ground-state energies. There is hope therefore that a microscopic approach could reproduce the comprehensive set of fission observables with some reasonable accuracy $(\sim 20-30 \%)$ in the short term.

This work performed under the auspices of the U.S. Department of Energy by Lawrence Livermore National Laboratory under Contract DEAC52-07NA27344.

\section{References}

1. W. Younes and D. Gogny, Phys. Rev. C 80, 054313 (2009).

2. W. Younes and D. Gogny, in Proceedings of the Fourth International Workshop on Nuclear Fission and Fission Product Spectroscopy, Cadarache, France 2009 (American Institute of Physics, New York, 2009), p. 3.

3. W. Younes and D. Gogny, Phys. Rev. Lett. 107, 132501 (2011).

4. J. Lennard-Jones, Proc. Roy. Soc. A 198, 14 (1949).

5. J.-F. Berger, M. Girod, D. Gogny, Nucl. Phys. A502, 85 (1989).

6. H. Goutte, J. F. Berger, P. Casoli, and D. Gogny, Phys. Rev. C 71, 024316 (2005).

7. R. Bernard, H. Goutte, D. Gogny, and W. Younes, Phys. Rev. C 84, 044308 (2011).

8. Ch. Straede, C. Budtz-Jorgensen, and H.-H. Knitter, Nucl. Phys. A462, 85 (1987).

9. P. Schillebeeckx, C. Wagemans, A. J. Deruytter, and R. Barthélémy, Nucl. Phys. A545, 623 (1992).

10. K.-H. Schmidt and B. Jurado, JEF/DOC 1423 (2011).

11. F. Gönnenwein, J. P. Bocquet, and R. Brissot, Proc. 17th Int. Symp. Nucl. Phys., Gaussig, GDR, 1987, 129 (ZfK-646).

12. S. Goriely, S. Hilaire, M. Girod, and S. Peru, Phys. Rev. Lett. 102, 242501 (2009). 